\title{
Treatment of tungiasis with a two- component dimeticone: a comparison between moistening the whole foot and directly targeting the embedded sand fleas
}

Per Nordin ${ }^{1,5^{*}}$ (D), Marlene Thielecke ${ }^{2}$, Nicholas Ngomi ${ }^{3}$, George Mukone Mudanga ${ }^{4}$, Ingela Krantz ${ }^{1}$ and Hermann Feldmeier ${ }^{2}$

\begin{abstract}
Background: Tungiasis (sand flea disease) is caused by the penetration of female sand fleas (Tunga penetrans, Siphonaptera) into the skin. It belongs to the neglected tropical diseases and is prevalent in South America, the Caribbean and sub-Saharan Africa. Tungiasis predominantly affects marginalized populations and resource-poor communities in both urban and rural areas. In the endemic areas, patients do not have access to an effective and safe treatment. A proof-of-principle study in rural Kenya has shown that the application of a two-component dimeticone (NYDA ${ }^{\oplus}$ ) which is a mixture of two low viscosity silicone oils caused almost $80 \%$ of the embedded sand fleas to lose their viability within 7 days.

Methods: In this study we compared the efficacy of two distinct modes of application of NYDA ${ }^{\oplus}$; one targeted application to the area where the parasite protrudes through the skin and one comprehensive application to the whole foot.

Results: Independent of the two modes of application, the dimeticone caused more than 95\% of embedded sand fleas to lose all signs of viability within 7 days. The targeted application killed embedded sand fleas more rapidly compared to when the whole foot was covered. The proportion of viable lesions at day two were 7.0 versus $23.4 \%$ $(p<0.01)$ and at day five 3.9 versus $12.5 \%(p<0.02)$.
\end{abstract}

Conclusions: Our findings suggest that the dimeticone could provide a safe and effective treatment for tungiasis in areas with difficult access to health care.

Trial registration: ISRCTN ISRCTN74306878

Keywords: Tungiasis, Treatment, Dimeticone, Public health

\section{Background}

Tungiasis (sand flea disease) belongs to the family of neglected tropical diseases and is prevalent in South America, the Caribbean and sub-Saharan Africa [1]. It is caused by penetration of female sand fleas (Tunga penetrans) into the skin and the ensuing inflammatory response

\footnotetext{
* Correspondence: per.nordin@skaraborg-institute.se

${ }^{1}$ The Skaraborg Institute for Research and Development, Stationsgatan 12 54130 Skövde, Sweden

${ }^{5}$ Epidemiology and Global Health, Department of Public Health and Clinical Medicine, Umeå University, Umeå, Sweden

Full list of author information is available at the end of the article
}

[2]. The inflammation is intensified by an almost unavoidable bacterial super-infection [3]. Just about all of the lesions are found in the feet $[4,5]$. The consequences of sand flea disease are debilitating, eventually leading to chronic morbidity with impaired mobility and quality of life $[6,7]$.

The prevalence of tungiasis varies between settings; prevalences up to $60 \%$ have been reported in various populations with up to $80 \%$ in children [8-11]. Children and the elderly are more likely to develop severe disease $[4,11,12]$. Tungiasis predominantly affects marginalized populations and people living in resource-poor communities in both urban and rural areas $[8,10,13-15]$. 
Various drugs have been examined for their efficacy against embedded sand fleas in humans. Randomized controlled trials using topical or oral administration of antihelminthic drugs such as metrifonate, thiabendazole or ivermectin showed little or no efficacy at all [16-19].

The only treatment option patients in the endemic areas have is to try to kill embedded sand fleas chemically or mechanically. People often apply toxic substances such as kerosene, used engine oil or household insecticides. Alternatively, the lesions are manipulated with sharp instruments such as needles, safety pins, razor blades or thorns, a health risk by itself. Such practices can also cause additional bacterial super-infections or transmit viral pathogens such as HBV, HCV and HIV [7].

The last three abdominal segments of an embedded sand flea form a miniature cone through which the parasite remains in contact with the environment through an opening in the skin of about $250 \mu \mathrm{m}$. Through this opening, the female sand flea takes up oxygen, expels eggs, defecates and gets fertilized. The abdominal cone protrudes through the skin and has been identified as a target for drug treatment [20,21]. As the skin around the abdominal cone is painful, patients usually know exactly how to localize an embedded sand flea.

A proof-of-principle study in rural Kenya has shown that the application of a two-component dimeticone ( $\left.\mathrm{NYDA}^{\circ}\right)$ to the skin of the feet, repeated two times within 5 min, kills almost $80 \%$ of the embedded fleas within 7 days [21]. Furthermore, assessments of lesion morphology indicate that normal development was interrupted in those parasites not killed: the female fleas became unable to produce and/or expel eggs. Lesion-associated inflammation significantly decreased within 7 days after application of dimeticone [21].

$\mathrm{NYDA}^{\circ}$ contains two dimeticones or silicone oils with different viscosities and a high creeping property. It is commercialized as a medical device for the treatment of head lice infestation in many European countries $[22,23]$. Its mode of action is purely physical [24].

In this study, we compared the efficacy of two distinct modes of application of $\mathrm{NYDA}^{\circ}$ : one targeted application to the area where the abdominal cone of the parasite protrudes through the skin and one general application to the whole foot.

The rationale for the targeted application was twofold: first, to minimize the volume of the dimeticone and, second, to direct the dimeticone to where it should act, namely the vital organs of the parasite located inside the abdominal cone. By consequence, a targeted application of the dimeticone should lead to a more rapid death of an embedded sand flea.

\section{Methods}

The study took place from the end of February till the end of March 2014, i.e. during the end of the dry season, when transmission of tungiasis peaks.

\section{Study area}

The study was conducted in eight primary schools in Bugiri district, Bulidha sub-county, eastern Uganda. The schools were located in the following villages: Makoma, Isaka Bisolo, Kibuye, Businda, Busakira, Nakawa and Wakawaka.

\section{Study population}

Sixty children aged 5 to 12 years selected from eight primary schools were included in the study. The number of children sampled from each school was based on the school and class size as well as on the organizational convenience. The number of children enrolled per school varied from 3 to 21 .

Children from classes one to six present at the day of the investigation were eligible for the study provided they had at least three viable sand flea lesions on each foot (stage 2 and/or 3 according to the Fortaleza classification [25]) as evaluated by a rapid assessment method [26].

They should furthermore not show clinical symptoms requiring immediate medical attention such as abscesses, ulcers or intense pain. When multiple embedded sand fleas of stage 2 or 3 were present, only lesions which could be clearly distinguished from one another were included in the study.

Lesions were chosen so that their location made it possible to use the handheld digital microscope, such as at the toe, the sole or the rim of the foot. Each lesion was photographed, and its location and stage was noted in the patient's record. The number of lesions included in the study was limited to three per foot and were followed up at regular intervals for 7 days (Fig. 1).

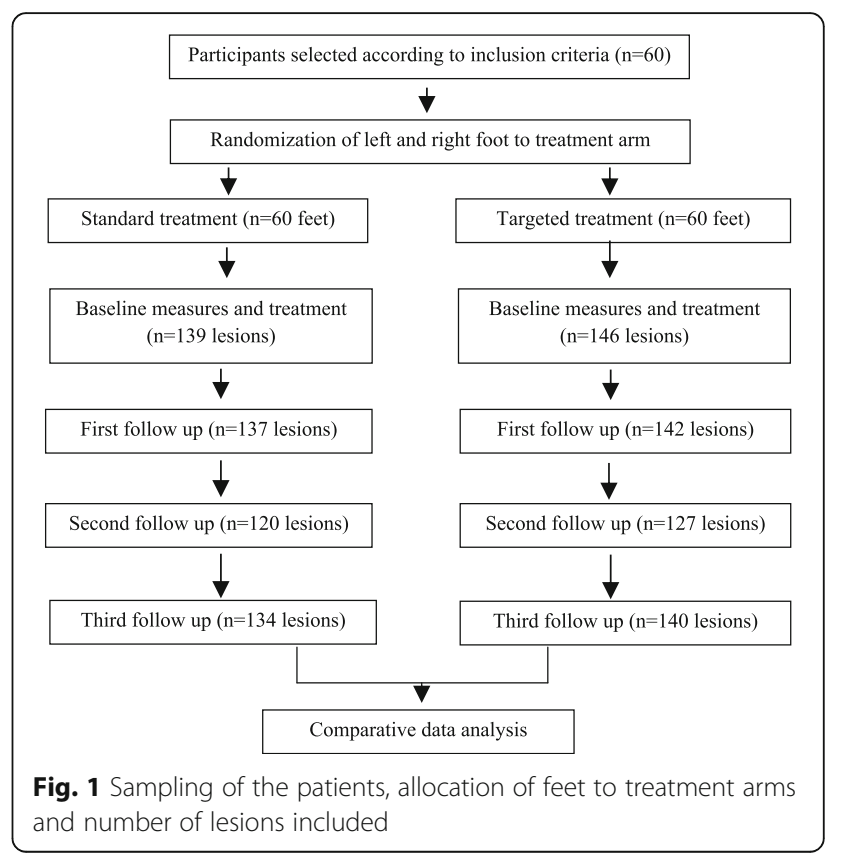




\section{Study design}

Two topical regimens of the two-component dimeticone (NYDA ${ }^{\circ}$ ) (Pohl-Boskamp GmbH \& Co. KG, Hohenlockstedt, Germany) were randomly allocated to either the left or the right foot of the patient. Before the application of dimeticone, both feet were washed with water and soap and dried with a clean towel.

The regimens were labeled 'whole foot treatment' and 'targeted treatment'. Whole foot treatment meant that dimeticone was applied to the skin of the foot up to the ankle, as previously described [21]. The application was stopped when the skin became shiny, indicating that it was wetted with the dimeticone. This procedure was repeated three times within $10 \mathrm{~min}$ and required 2 to $5 \mathrm{ml}$ depending on the size of the foot.

Targeted treatment meant that dimeticone was aspired into a 5-ml syringe to which a flexible tube was mounted. Three drops were applied to the area where the parasite's abdominal cone protruded through the skin. One drop corresponds to approximately $50 \mu \mathrm{l}$ of dimeticone. This procedure was repeated three times within $10 \mathrm{~min}$ to ensure that a maximum amount of dimeticone entered into the abdominal cone of the parasite within a short period of time. This required approximately $450 \mu \mathrm{l}$ per embedded sand flea. In both groups, the dimeticone was only applied at baseline.

For each patient, demographic data as well as baseline parasitological measurements were conducted as previously described [21, 25, 27]. Staging was performed according to the Fortaleza Classification.

- Stage I: penetrating sand flea

- Stage II: brownish-black dot with a diameter of 1-2 mm

- Stage III: circular yellow-white watch glass-like patch with a diameter of 3-10 $\mathrm{mm}$ and with a central black dot

- Stage IV: brownish-black crust with or without surrounding necrosis

Stage I to III are viable sand fleas; in stage IV, the parasite is dying or already dead [25].

Using a digital handheld microscope (dnt DigiMicro Mobile 5-megapixel-handheld-microscope, ITEZ, Hong Kong, China) viability signs (expulsion of eggs, excretion of faeces threads, excretion of liquid, pulsations/ contractions) were recorded as present or not.

\section{Outcome measures}

All data were collected by the same investigator at baseline and during a 7-day follow-up: day two, day five and day seven.

Two major outcome measures were defined: the viability of the embedded sand flea and the intensity of the local inflammation. The primary outcome measure was the viability of the embedded sand flea according to the Fortaleza Classification [25]. An embedded sand flea was considered to be dead, if none of the four viability signs was detected during $15 \mathrm{~min}$ of observation by the digital handheld microscope on two consecutive follow-up examinations [20]. A secondary outcome measure was the intensity of the local inflammation, as assessed semiquantitatively by an inflammation score [21]. Lesions manipulated by the patient or the caregiver were also documented.

Another set of outcome measures were based on visual scales depicting how the impact of tungiasis was perceived by the patient. The scales consist of a series of simple pictures illustrating itching, pain, itchingrelated sleep disturbance, pain-related sleep disturbance and mobility impairment as perceived by the patient. The patient was asked to classify the degree of each complaint by pointing to the corresponding picture. Zero meant no complaint at all, 1 = little complaint, $2=$ moderate complaint, $3=$ severe complaint and $4=$ very severe complaint. These outcome measures encompass both feet, since it was considered impossible for the participants to discern the impact of embedded sand fleas separately for each foot.

\section{Statistical methods}

The sample size of circa 140 lesions per treatment group is based on a $15 \%$ difference in treatment effect, a power of $90 \%$ and a significance level of $5 \%$. The assumption was based on our previous findings [21]. Fisher's exact test was used to compare proportions, and the Kruskal-Wallis test was used to analyze the inflammation score and self-reported tungiasis-related characteristics. A relationship was considered statistically significant when a $p$ value was less than $5 \%$. All presented confidence interval (CI) have a confidence level set at $95 \%$.

\section{Results}

\section{Baseline}

At baseline, the two treatment groups displayed similar characteristics concerning the distribution of lesion types (Table 1).

The difference between the number of lesions found on the two feet for the same individual were never larger than 12 in all cases except one. In this particular case, the foot intended for whole foot treatment had 75 lesions and the foot intended for targeted treatment only nine lesions. The distribution of differences in number of lesions between the paired feet, i.e. feet on the same individual are considered a pair, showed a mean of 0.1 together with a standard deviation of 9.7, and the related median had a value of 0 . The average numbers of viable 
Table 1 Types of lesions at baseline in the two treatment groups

\begin{tabular}{|c|c|c|c|c|}
\hline \multirow[b]{2}{*}{ Lesion type $^{a}$} & \multicolumn{2}{|c|}{ Whole foot treatment } & \multicolumn{2}{|c|}{ Targeted treatment } \\
\hline & Median & (Min-max) & Median & (Min-max) \\
\hline Viable lesion & 4 & $(1-75)$ & 5 & $(1-50)$ \\
\hline Non-viable lesion & 4 & $(0-30)$ & 4 & $(0-30)$ \\
\hline Manipulated lesion & 1 & $(0-12)$ & 1 & $(0-15)$ \\
\hline All lesions & 9 & $(1-94)$ & 10 & $(2-70)$ \\
\hline
\end{tabular}

${ }^{a}$ According to definition in subjects and methods

lesions were close to seven per foot with a substantial variation in both treatment groups. The two groups also displayed a similar distribution of frequencies across the range of the parasites' developmental stages (Table 2).

\section{During and after treatment}

The outcome measured as the loss of viability of embedded sand fleas after the application of the dimeticone is shown in Fig. 2.

At the first follow-up, 2 days after treatment, the number of viable parasites decreased significantly in both groups (whole foot treatment $p<0.001$; targeted treatment $p<0.001)$. The loss of viability was higher in the targeted treatment group $(p<0.001)$. At the second follow-up, after 5 days, the loss of viability was still higher in the targeted treatment group compared to the whole foot treatment group $(p<0.02)$. After 7 days, the reduction of the number of viable sand fleas was similar in both groups: $95 \%$ (CI 92; 99) of the parasites in the whole foot treatment group had lost all signs of viability and $97 \%$ (CI 94; 99) in the targeted treatment group (Table 3). Furthermore, in both groups, sand fleas which remained viable did not expel eggs during the 7 days. Whether lesions were in stage 2 or 3 at baseline had no impact on the efficacy of either treatment (Table 3).

The inflammation scores and the visual scale measurements showed significant reductions between baseline and day seven (Table 4).

After completion of the study (day 7), the medians of all visual scales had decreased to 0 .

Table 2 Number of viable lesions per developmental stage of lesions in the two treatment groups at baseline

\begin{tabular}{lll}
\hline Stage $^{\text {a }}$ & Whole foot treatment & Targeted treatment \\
\hline $2 \mathrm{a}$ & 30 & 39 \\
$\mathrm{2b}$ & 96 & 93 \\
$3 \mathrm{a}$ & 12 & 12 \\
$3 \mathrm{~b}$ & 1 & 2 \\
Total number & 139 & 146
\end{tabular}

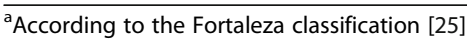

\section{Discussion}

Tungiasis is endemic in resource-poor populations in many countries of sub-Saharan Africa $(8,10,13,14,15)$. It is associated with important morbidity, and children and the elderly carry the highest disease burden $(4,11$, 12). Nonetheless, hitherto there is no approved treatment. In Kenya, the Ministry of Health recommends bathing the feet for $10 \mathrm{~min}$ in $\mathrm{KMnO} 4$, an approach with a rather low efficacy [21]. Besides, $\mathrm{KMnO} 4$ stains the skin in deep purple. This makes the treatment visible for everyone and the patient vulnerable to ridiculosity [21]. Hence, there is an urgent need for a safe and effective treatment of tungiasis.

The study showed that with the targeted application of the dimeticone, parasites were killed more rapidly and that 2 days after the topical application, only $7 \%$ of the parasites remained viable (Table 3). After 7 days, though, in both treatment groups, $>95 \%$ of the embedded sand fleas had lost all viability signs. A more rapid death of the parasites is an advantage, because inflammation resolves as soon as an embedded sand flea has died (Thielecke $M$, unpublished observation 2014, [28]).

It is likely that by a repeated targeted application, more dimeticone crept into the abdominal cone per unit of time compared to when the skin of the whole foot is wetted and that this resulted in a rapid death.

Actually, when looking at the abdominal cone with the digital microscope, one could see how the dimeticone creeps into the opening in the skin and then disappears, as it spreads to the microscopic surfaces located within the abdominal cone. A similar observation has been made when dimeticone is applied to free running insects such as head lice and crickets [23].

Seven days after the application of the dimeticone, $98 \%$ of the parasites had lost all their viability signs in the targeted treatment group and $95.5 \%$ in the whole foot treatment group. Only a few sand fleas withstand the treatment, but these were not observed to expel eggs during the remainder of the study, indicating that the dimeticone had abrogated the normal development of the female sand flea as observed previously [21]. The implication of this finding is that a scaled-up treatment program could have an effect on the transmission.

Tungiasis-related symptoms decreased rapidly, when the sand fleas lost their viability by the dimeticone. This was visible both as indicated by the inflammation score assessed by the investigator, as well as by the visual scales as expressed by the patients themselves (Table 4). Notably, the median became 07 days after treatment in all visual scales. One week after treatment, healing was evident as seen by the decrease in the inflammation score as well as the visual scales confirming a previous finding [21]. 


\section{Parasite viability development}

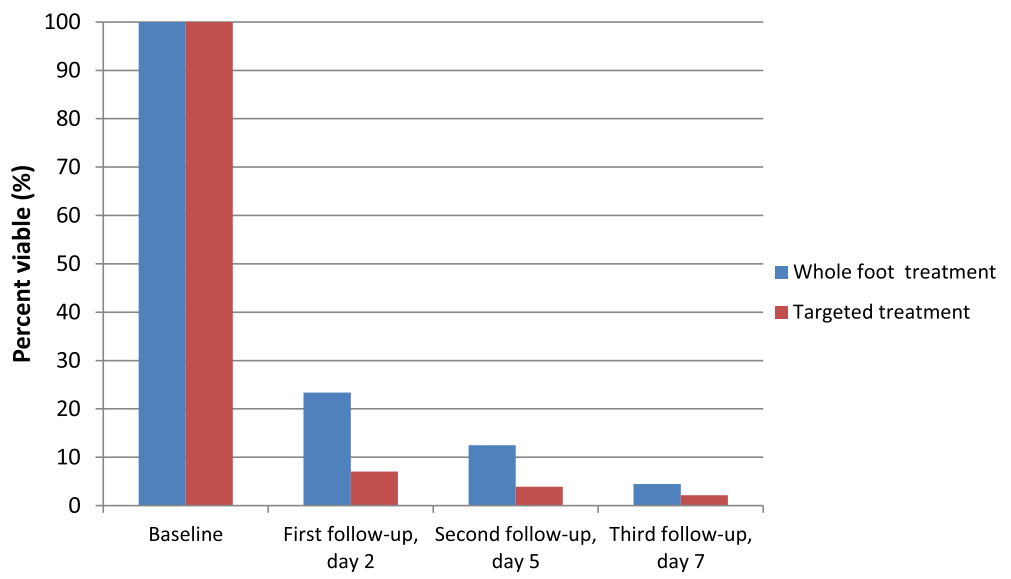

Fig. 2 Decline of parasite viability from baseline with treatment for each group through the three follow-up examinations at day 2, day 5 and day 7

During a previous study, we have observed that the inflammation around the embedded sand fleas increase when they continue to grow and intensify their metabolic activity in stages 2 and 3 [27]. The inflammation recedes, however, when the parasites develop from stage 3 to 4 [25]. In these stages, the signs of viability slowly disappear and eventually the parasites die.

The efficacy of the whole foot treatment here was higher compared to the proof-of-principle study in Kenya where dimeticone applied to the feet up to ankles killed $78 \%$ of the embedded sand fleas within 7 days [21]. Apart from the difficulty of achieving a standardized application procedure giving a precise dosage for each foot, there are also biological explanations for this difference. Rapid penetration of the dimeticone into the last abdominal segments of the embedded sand flea may depend on how deep a parasite is located in the skin. The thicker the corneal layer of the skin, the longer the dimeticone will need to reach the stratum of the epidermis in which the parasites are located. In addition, only a part of the dimeticone will be absorbed by a rough and thickened epidermis. The children in Kenya did not use shoes at all, whereas the children in Uganda, at least partially, had sandals or flip-flops. Thus, the higher efficacy of the dimeticone in this study might reflect a thinner and smoother corneal layer of the feet of the Ugandan participants.

Table 3 Viability of embedded sand fleas in the treatment groups at baseline and the subsequent follow-ups ${ }^{a}$

\begin{tabular}{|c|c|c|c|c|c|c|c|c|}
\hline & \multirow[b]{2}{*}{ Lesion stage $^{b}$} & \multicolumn{3}{|c|}{ Whole foot treatment } & \multicolumn{4}{|c|}{ Targeted Treatment } \\
\hline & & No. viable & No. non-viable & Viable (\%) & No. viable & No. non-viable & Viable (\%) & $p$ value \\
\hline \multirow[t]{3}{*}{ Baseline (day 0) } & All lesions & 139 & 0 & 100 & 146 & 0 & 100 & n.a. \\
\hline & stage 2 & 126 & 0 & 100 & 132 & 0 & 100 & n.a. \\
\hline & stage 3 & 13 & 0 & 100 & 14 & 0 & 100 & n.a. \\
\hline \multirow[t]{3}{*}{ First follow-up (day 2) } & All lesions & 32 & 105 & 23.4 & 10 & 132 & 7.0 & $<0.001$ \\
\hline & stage 2 & 31 & 92 & 25.2 & 8 & 116 & 6.5 & $<0.001$ \\
\hline & stage 3 & 1 & 13 & 7.1 & 2 & 16 & 11.1 & 0.600 \\
\hline \multirow[t]{3}{*}{ Second follow-up (day 5) } & All lesions & 15 & 105 & 12.5 & 5 & 122 & 3.9 & 0.018 \\
\hline & stage 2 & 14 & 95 & 12.8 & 5 & 109 & 4.4 & 0.030 \\
\hline & stage 3 & 1 & 10 & 9.1 & 0 & 13 & 0 & 0.458 \\
\hline \multirow[t]{3}{*}{ Third follow-up (day 7) } & All lesions & 6 & 128 & 4.5 & 3 & 137 & 2.1 & 0.326 \\
\hline & stage 2 & 5 & 115 & 4.2 & 3 & 120 & 2.4 & 0.496 \\
\hline & stage 3 & 1 & 13 & 7.1 & 0 & 17 & 0.0 & 0.452 \\
\hline
\end{tabular}

n.a. not applicable

${ }^{a}$ Not all participants and all lesions could be examined at every occasion, which explains differences in the numbers of examined lesions during follow-ups

${ }^{\mathrm{b}}$ According to the Fortaleza classification [25] 
Table 4 Secondary outcome measures at baseline and at day seven for both feet combined

\begin{tabular}{|c|c|c|c|c|c|c|c|c|c|c|c|}
\hline \multirow[b]{2}{*}{ Outcome } & \multicolumn{6}{|c|}{ Baseline } & \multicolumn{5}{|l|}{ Day 7} \\
\hline & $\mathrm{N}$ & Median & $\mathrm{IQR}^{\mathrm{a}}$ & Min & Max & $\mathrm{N}$ & Median & $I Q R^{a}$ & Min & Max & $p$ value $^{b}$ \\
\hline Inflammation score & 56 & 4.3 & 3.4 & 1 & 22 & 57 & 0.5 & 1.5 & 0 & 12 & $<0.001$ \\
\hline \multicolumn{12}{|l|}{ Visual scales } \\
\hline Intensity of spontaneous pain & 60 & 3 & 1 & 1 & 4 & 60 & 0 & 1 & 0 & 4 & $<0.001$ \\
\hline Intensity of itching & 60 & 3 & 1 & 1 & 4 & 60 & 0 & 1 & 0 & 3 & $<0.001$ \\
\hline Itch-related sleep disturbance & 60 & 2 & 1 & 1 & 4 & 60 & 0 & 1 & 0 & 4 & $<0.001$ \\
\hline Pain-related sleep disturbance & 60 & 2 & 1 & 1 & 4 & 60 & 0 & 0 & 0 & 3 & $<0.001$ \\
\hline Degree of mobility impairment & 52 & 2 & 1 & 1 & 4 & 59 & 0 & 1 & 0 & 4 & $<0.001$ \\
\hline
\end{tabular}

Interquartile range

${ }^{\text {b} K r u s k a l l-W a l l i s ~ t e s t ~}$

Lesions localized to the tip of the toes, the sole and the rim of the foot were deliberately chosen so that the handheld digital microscope easily could be applied in order to assess viability signs with a higher degree of precision. At those selected sites, it can be suspected that the dimeticone might be targeted more precisely and/or penetrate the parasite more rapidly. No such selections were made in the Kenyan study. There, the included lesions were also located under the nail, under thick crusts of the corneal layer or in necrotic tissue areas into which the dimeticone cannot penetrate easily. A difference in penetration efficiency could thus possibly also explain the differing results.

The number of lesions varied considerably within the group of included children (Table 2). When the number of lesions are more than three, they often occur in clusters [29] making it difficult to distinguish the characteristics of an individual lesion, which also impairs assessments of morphological changes and the lesionassociated inflammation [21]. We, therefore, deliberately limited the number of included lesions to three per foot.

The results of this study are based on highly controlled and monitored procedures. This means one ought to be cautious when considering the true effectiveness of treatment of tungiasis with dimeticone under field conditions. Based on the average required volume of dimeticone for treatment of the whole foot and what is used in the targeted treatment, we find that the targeted application is more parsimonious as long as the number of viable embedded sand fleas does not exceed seven lesions per foot. Hence, in severe cases where individuals suffer from dozens of embedded viable sand fleas, the whole foot treatment should be more cost-effective and also more practicable.

\section{Conclusions}

The application of a mixture of a two-component dimeticone $\left(\mathrm{NYDA}^{\circ}\right)$ caused more than $95 \%$ of embedded sand fleas to lose all defined signs of viability within 7 days. The targeted topical application worked faster compared to when the whole foot was covered. Our findings suggest that the dimeticone could provide a safe and effective treatment for tungiasis in areas with difficult access to health care.

\section{Abbreviations}

Cl: Confidence interval; HBV: Hepatitis B virus; HCV: Hepatitis C virus: HIV: Human immunodeficiency virus; ISRCTN: International standard randomised controlled trials number; WHO: World Health Organization

\section{Acknowledgements}

The authors would like to thank the Ministry of Health, Uganda, for giving the study a go ahead; the District Health Officer Bugiri for welcoming and supporting the team into the district; and the headmasters, teachers and pupils from the participating primary schools for their support during the field work.

\section{Funding}

The study was funded by German Doctors e. V., registered charity, Bonn, Germany.

\section{Availability of data and materials}

The datasets generated and/or analyzed during the current study are not publicly available due to the fact that they in their raw form contain information that theoretically could make it possible to identify an individual. The data can be made available from the corresponding author on reasonable request, but to protect the integrity of the examined individuals and based on the nature of the request, we reserve the right to remove such data used to identify particular individuals.

\section{Authors' contributions}

$\mathrm{HF}, \mathrm{PN}$ and MT conceived and designed the experiments. GM and NN performed the field work. PN, HF and IK analyzed the data. PN, MT, HF and IK wrote the paper. All authors contributed to the critical revision of the manuscript for important intellectual content. All authors read and approved the final manuscript

\section{Competing interests}

HF has received lecture fees from Pohl-Boskamp GmbH and Co KG, Hohenlockstedt, Germany, the producer of NYDA. The company had no role in the design, execution or interpretation of the study. The other authors do not have any conflicts of interest to declare.

\section{Consent for publication}

Not applicable.

\section{Ethics approval and consent to participate}

The study was registered in the Current Controlled Trials 2014 (ISRCTN74306878) and approved by the Ethics Committee of Makerere University College of Health Sciences, School of Public Health, Makerere, Uganda, (Higher Degrees Research and Ethics Committee Protocol No. 157). Written consent was signed, either by signature or fingerprint, by the 
guardians of the participants after they were informed about the trial in the local language Lusoga. The headmaster of the respective schools acted as a witness. At the end of the study, any remaining viable sand fleas were treated with a targeted application of dimeticone. All participants received a new pair of closed shoes, since wearing such shoes has been shown to reduce the incidence of sand flea disease [25]. Children not eligible for the study because of symptoms, such as intense pain, abscesses or ulcers, which required immediate treatment, were referred to the nearest Community Health Center.

\section{Endnotes}

Not applicable.

\section{Publisher's Note}

Springer Nature remains neutral with regard to jurisdictional claims in published maps and institutional affiliations.

\section{Author details}

${ }^{1}$ The Skaraborg Institute for Research and Development, Stationsgatan 12, 54130 Skövde, Sweden. ${ }^{2}$ Institute of Microbiology and Hygiene, Campus Benjamin Franklin, Charité University Medicine, Berlin, Germany. ${ }^{3}$ African Population and Health Research Center, Nairobi, Kenya. ${ }^{4}$ Department of National Disease Control, Ministry of Health, Kampala, Uganda.

${ }^{5}$ Epidemiology and Global Health, Department of Public Health and Clinical Medicine, Umeå University, Umeå, Sweden.

Received: 8 November 2016 Accepted: 2 March 2017 Published online: 10 March 2017

\section{References}

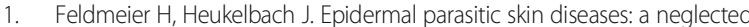
category of poverty-associated plagues. Bull World Health Org. 2009;87:152-9. doi:10.2471/BLT.07.047308.

2. Feldmeier $H$, Heukelbach J, Ugbomoiko US, Sentongo E, Mbabazi $P$, et al. Tungiasis - a neglected disease with many challenges for global public health. PLoS Negl Trop Dis. 2014;8(10):e3133. doi:10.1371/journal.pntd. 0003133.

3. Feldmeier $\mathrm{H}$, Heukelbach J, Eisele M, Carvalho CBM. Bacterial superinfection in human tungiasis. Trop Med Int Health. 2002;7:559-64.

4. Muehlen $\mathrm{M}$, Heukelbach J, Wilcke $\mathrm{T}$, Winter $\mathrm{B}$, Mehlhorn $\mathrm{H}$, Feldmeier $\mathrm{H}$. Investigations on the biology, epidemiology, pathology and control of Tunga penetrans in Brazil: II. Prevalence, parasite load and topographic distribution of lesions in the population of a traditional fishing village. Parasitol Res. 2003:90:449-55.

5. Heukelbach J, Wilcke T, Eisele M, Feldmeier H. Ectopical localization of tungiasis. Am J Trop Med Hyg. 2002:67:214-6.

6. Feldmeier $\mathrm{H}$, Keysers A. Tungiasis - a Janus-faced parasitic skin disease. Trav Med Infect Dis. 2013:11:357-65.

7. Feldmeier $\mathrm{H}$, Sentongo E, Krantz I. Tungiasis (sand flea disease): a parasitic disease with intriguing challenges for public health. Eur J Clin Microbiol Infect Dis. 2012;32:19-26.

8. Chadee DD. Tungiasis among five communities in south-western Trinidad, West-Indies. Ann Trop Med Parasitol. 1998:92(1):107-13.

9. Nte AR, Eke FU. Jigger infestation in children in a rural area of Rivers State of Nigeria. West Afr J Med. 1995;14(1):56-8.

10. Ratovonjato J, Randriambelosoa J, Robert V. Tunga penetrans (Insecta, Siphonaptera, Tungidae) à Madagascar: une nuisance négligée. Rev Med Vet. 2008;11:551-6

11. Wilcke T, Heukelbach J, Moura RSC, Kerr-Pontes LRS, Feldmeier H. High prevalence of tungiasis in a poor neighbourhood in Fortaleza, Northeast Brazil. Acta Trop. 2002;83:255-8.

12. Ariza L, Seidenschwang M, Buckendahl J, Gomide M, Feldmeier $H$, et al. Tungiasis: a neglected disease causing severe morbidity in a shantytown in Fortaleza, State of Ceará. Rev Soc Bras Med Trop. 2007:40:63-7.

13. Heukelbach J, Costa AML, Wilcke T, Mencke N, Feldmeier H. The animal reservoir of Tunga penetrans in severely affected communities of north-east Brazil. Med Vet Entomol. 2004;18:329-35.

14. Heukelbach J, Oliveira F, Hesse G, Feldmeier H. Tungiasis: a neglected health problem of poor communities. Trop Med Int Health. 2001;6:267-72.
15. Muehlen M, Feldmeier $\mathrm{H}$, Wilcke T, Winter B, Heukelbach J. Identifying risk factor for tungiasis and heavy infestation in a resource-poor community in northeast Brazil. Trans R Soc Trop Med Hyg. 2006;100:371-80.

16. Heukelbach J, Eisele M, Jackson A, Feldmeier H. Topical treatment of tungiasis: a randomized, controlled trial. Ann Trop Med Parasitol. 2003;97:743-9.

17. Heukelbach J, Franck S, Feldmeier H. Therapy of tungiasis: a double-binded randomized controlled trial with oral ivermectin. Mem Inst Oswaldo Cruz. 2004:99:873-6.

18. Ahadi Kenya Trust. The Jigger Menace in Kenya Report Volume 2. 2010 Available: http://www.jigger-ahadi.org/anti_jigger_magazine_year_2 \%20final.pdf. Accessed 15 June 2016.

19. Heukelbach J, Wilcke T, Winter B, de Oliveira FA, Moura RCS, Harms G, Liesenfeld $\mathrm{O}$, Feldmeier $\mathrm{H}$. Efficacy of ivermectin in a patient population concomitantly infected with intestinal helminths and ectoparasites. Arzneim-Forsch/Drug Res. 2004;54(7):416-21.

20. Thielecke $M$, Feldmeier $H$. The fate of the embedded virgin sand flea Tunga penetrans: hypothesis, self-experimentation and photographic sequence. Trav Med Infect Dis. 2013;11(6):440-3

21. Thielecke M, Nordin P, Ngomi N, Feldmeier H. Treatment of tungiasis with dimeticone: a proof-of-principle study in rural Kenya. PLoS Negl Trop Dis. 2014:8(7):e3058. doi:10.1371/journal.pntd.0003058.

22. Feldmeier $\mathrm{H}$. Treatment of pediculosis capitis: a critical appraisal of the current literature. Am J Clin Dermatol. 2014:15:401-12.

23. Richling I, Böckeler W. Lethal effects of treatment with a special dimeticone formula on head lice and house crickets (Orthoptera, Ensifera: Acheta domestica and Anoplura, Phthiraptera: Pediculus humanus). Drug Res. 2008; 58(5):248-54. doi:10.1055/s-0031-1296501.

24. Burgess IF. The mode of action of dimeticone $4 \%$ lotion against head lice. Pediculus capitis. 2009;9(3). doi:10.1186/1471-2210-9-3.

25. Eisele M, Heukelbach J, Van Marck E, Mehlhorn H, Meckes O, et al. Investigations on the biology, epidemiology, pathology and control of Tunga penetrans in Brazil: I. Natural history of tungiasis in man. Parasitol Res. 2003;90:87-99.

26. Ariza L, Wilcke T, Jackson A, Gomide M, Ugbomoiko US, Feldmeier $H$, et al. A simple method for rapid community assessment of tungiasis. Trop Med Int Hlth. 2010;15(7):856-64.

27. Thielecke M, Raharisolo C, Ramarokoto $\mathrm{CH}$, Rogier C, Randriamanantena H, Stauss-Grabo M, Richard V, Feldmeier $\mathrm{H}$. Prevention of tungiasis and tungiasis-associated morbidity: a randomized, controlled field study in rural Madagascar. Am J Trop Med Hyg. 2012;89:932-6.

28. Thielecke M, Raharimanga V, Stauss-Grabo M, Rogier C, Richard V, Feldmeier H. Regression of severe tungiasis-associated morbidity after prevention of re-infestation: a case series from rural Madagascar. Am J Trop Med Hyg. 2013;89:932-6.

29. Feldmeier H, Eisele M, Heukelbach J, Saboia Moura RC. Severe tungiasis in underprivileged communities: case series from Brazil. Emerg Infect Dis. 2003;9:949-55.

\section{Submit your next manuscript to BioMed Central and we will help you at every step:}

- We accept pre-submission inquiries

- Our selector tool helps you to find the most relevant journal

- We provide round the clock customer support

- Convenient online submission

- Thorough peer review

- Inclusion in PubMed and all major indexing services

- Maximum visibility for your research

Submit your manuscript at www.biomedcentral.com/submit
C) Biomed Central 